\title{
Analysis of Energy Reception Characteristics of Solar Aircraft in the Tropics of Cancer and Capricorn
}

\author{
Kangwen Sun ${ }^{1}$, Jiaqi Dong ${ }^{1}$, Xiaobo Sun ${ }^{2, *}$, Ziying Zhang ${ }^{1}$, Mou Sun ${ }^{1}$ and Jian Zhang ${ }^{3}$ \\ ${ }^{1}$ School of Aeronautic Science and Engineering, Beihang University, Beijing 100191, China \\ ${ }^{2}$ China Construction Eighth Engineering Division. CORP.LTD, Shanghai 200135, China \\ ${ }^{3}$ Forging Factory, North General Power Group Co., Ltd, 037000 Datong, Shanxi, China
}

\begin{abstract}
This paper mainly aims at the energy reception characteristics of solar aircraft flying in the near space between the Tropics of Cancer and Capricorn. Based on the solar radiation intensity calculation model, the energy reception characteristics including maximum solar radiation intensity, daytime average solar radiation intensity, total solar radiation energy received per day on the unit area., and daytime hours of solar aircraft was calculated varying with date, longitude, and latitude. By analyzing the corresponding calculation results, it can provide some reference value for the design of solar aircraft flying in this area.
\end{abstract}

\section{Introduce}

Solar aircraft is a new type of aircraft which can realize High Altitude Long Endurance(HALE) flight ${ }^{[1]}$ and has an irreplaceable advantage compared with conventional aircraft ${ }^{[2]}$. It can convert solar radiant energy into electrical energy by photovoltaic conversion. Near space generally refers to the airspace $20-100 \mathrm{~km}$ from the ground, and it has a special strategic position because of the unique height and environmental conditions. Using the stable atmosphere of near space and the inexhaustible supply of solar energy, HALE solar aircraft has a wide application prospect in the tasks of environmental detection, regional communication, border monitoring, disaster warning and monitoring, high resolution, etc., and it is one of the hot and frontier fields of international research ${ }^{[3]}$. Solar aircraft uses solar energy as the main power source ${ }^{[1]}$. Therefore, the analysis of solar energy reception characteristics is the basis and necessary prerequisite for the study of such aircraft ${ }^{[4]}$. Due to the influence of earth-sun distance, time and latitude, the solar radiation received in the near space will change. On account of the existence of the obliquity of the ecliptic, sunlit point is always moving back and forth between the Tropics of Cancer and Capricorn, which causes the solar energy in this area is abundant ${ }^{[5,6]}$.

In view of this, in this paper, the energy reception characteristics of solar aircraft which flies in the near space between the Tropics of Cancer and Capricorn were analysed, with the change of time, latitude and longitude, in order to provide some reference value for the design of this kind of aircraft.

\section{Calculation model of solar radiation intensity ${ }^{[7]}$}

Assuming that the influence of cloud, moist, dust, etc. is ignored, the calculation model of solar irradiation intensity is as follows.

\subsection{Solar altitude}

The solar altitude is the angle between the incident ray and the horizontal plane, and it will vary with time and place. The solar altitude $\alpha$ can be calculated as follows:

$$
\sin \alpha=\sin \Phi \sin \delta+\cos \Phi \cos \delta \cos \omega
$$

Where: $\Phi$ - local latitude, $\delta$ - sun declination angle, $\omega$ - solar hour angle.

$$
\delta=23.45^{\circ} \sin \left[\frac{360^{\circ}\left(d_{n}+284\right)}{365}\right]
$$

Where: $d_{n}$ - date number in a year

$$
\begin{gathered}
\omega=\left(t_{\mathrm{t}}-12\right) \cdot 15^{\circ} \\
t_{\mathrm{t}}=t_{\mathrm{s}} \pm \frac{L-L_{\mathrm{S}}}{15}+\frac{e}{60} \\
e=9.87 \sin 2 B-7.53 \cos B-1.5 \sin B
\end{gathered}
$$

\footnotetext{
This work was supported by the National Natural Science Foundation of China under Grant No. 51775021, Key Laboratory of Aircraft Advanced Design Technology (Beihang University) Ministry of industry, the fifth youth top talent support program of Beihang University, and the Fundamental Research Funds for the Central Universities.
} 


$$
B=\frac{360\left(d_{n}-81\right)}{364}
$$

Where: $t_{\mathrm{t}}$ - true solar time, $t_{\mathrm{s}}$ - local standard time, $L$ local longitude, $L_{S}$ - the longitude of the local standard time, $e$ - the equation of time between true solar time and mean solar time.

\subsection{Eccentricity correction coefficient associated with earth-sun distance}

The sun is in a slightly eccentric position on the earth's elliptical orbit, so the distance between the sun and earth is not constant, and there are perihelion and aphelion. The distance between the sun and earth is usually corrected with the eccentricity correction coefficient $\xi_{0}$.

$$
\xi_{0}=\left(\frac{r_{0}}{r}\right)^{2}=1+0.033 \cos \left(\frac{2 \pi d_{n}}{365}\right)
$$

Where: $r_{0}$ - the average earth-sun distance, $r$ - the earth-sun distance of the observation point.

\subsection{Atmospheric transparency coefficient ${ }^{[8]}$}

The atmospheric transparency coefficient is the ratio of transmitted radiation to incident radiation through an air mass. Under the different weather conditions, the atmosphere thickness will be different. Considering the weather factors, the atmospheric transparency coefficient $P_{\mathrm{CO}}$ can be calculated as follows:

$$
P_{\mathrm{CO}}=0.56 \cdot k\left(e^{-0.56 A M h}+e^{-0.096 A M h}\right)
$$

Where: $k$ - weather influence factor, $A M h$ - highly modified air mass.

$$
\begin{gathered}
A M h=A M \cdot \eta_{h} \\
A M=\frac{1}{\sin \alpha} \\
\eta_{h}=\operatorname{real}\left(1-\frac{0.0065}{288} H\right)^{5.256}
\end{gathered}
$$

Where: $A M$ - air mass, $\eta_{h}$ - atmospheric height correction factor, real- the value is the real part of the formula, $H$ - height.

\subsection{Sunrise and sunset time ${ }^{[9]}$}

Sunrise time $t_{\mathrm{sr}}$ is the time when the sun appears on the horizon. Regardless of the curvature of the earth's surface and the refraction of the atmosphere to the light, it can be calculated as follows:

$$
t_{\mathrm{sr}}=\arccos (\tan \Phi \tan \delta) \cdot \frac{180}{15 \pi}
$$

The sunset time $t_{\mathrm{ss}}$ and the sunrise time $t_{\mathrm{sr}}$ are symmetric at 12 noon.

$$
t_{\mathrm{ss}}=24-t_{\mathrm{sr}}
$$

Daytime hours $t_{\text {day }}$ are the difference between the sunset time and the sunrise time.

$$
t_{\mathrm{day}}=t_{\mathrm{ss}}-t_{\mathrm{sr}}
$$

\subsection{Solar radiation intensity}

Considering the effects of seasons, earth-sun distances, height, and time, solar radiation intensity $I$ can be calculated as follows:

$$
I=I_{\mathrm{sc}} \cdot \xi_{0} \cdot P_{\mathrm{co}} \cdot \sin \alpha
$$

Where: $I_{\mathrm{sc}}-$ solar constant, refers to the received solar radiation energy at the boundary of the earth's atmosphere surface perpendicular to the light in unit area and unit time. In 1981, the eighth session of the world meteorological organization instrument and observation method committee defined the solar constant as 1367W/ $\mathrm{m}^{2}$.

\section{6 solar energy reception characteristics}

The intensity of solar radiation increases from sunrise to noon and then decreases before sunset. It can be reduced to a sine curve, and the highest point is obtained at noon, which is the true solar time $t_{\mathrm{t}}=12$.

Maximum solar radiation intensity at noon $I_{\max }$ can be calculated as follows:

$$
I_{\max }=I_{\mathrm{sc}} \cdot \xi_{0} \cdot P_{\mathrm{co}} \cdot \sin \alpha_{\left(t_{\mathrm{t}}=12\right)}
$$

The change function of solar radiation intensity $I$ with time $t$ during the day is:

$$
I=I_{\text {max }} \cdot \sin \left(\frac{t-t_{\mathrm{sr}}}{t_{\text {day }}}\right) * \pi
$$

Daytime average solar radiation intensity $I_{\text {level }}$ can be calculated as follows:

$$
\begin{gathered}
I_{\text {level }}=\frac{1}{t_{\text {day }}} \int_{t_{\mathrm{sr}}}^{t_{\mathrm{ss}}} I_{\max } \cdot \sin \left(\frac{t-t_{\mathrm{sr}}}{t_{\mathrm{day}}}\right) * \pi d t \\
=\frac{2 I_{\max }}{\pi}
\end{gathered}
$$

The total amount of solar radiation received per unit area $E_{\mathrm{s}}$ in one day is:

$$
E_{\mathrm{s}}=I_{\text {level }} \cdot t_{\text {day }}
$$

According to the above calculation model, this paper will analyse the variation of $I_{\max }, I, I_{\text {level }}, E_{\mathrm{s}}$ and $t_{\text {day }}$ with date, longitude and latitude. Relevant constant value parameters are chosen as shown in table 1.

Table 1. Parameters of solar irradiation strength calculation.

\begin{tabular}{|c|c|c|}
\hline Parameter & Value & Unit \\
\hline$L_{S}$ & 120 & $\circ$ \\
\hline$k$ & 0.85 & - \\
\hline$H$ & 20000 & $\mathrm{~m}$ \\
\hline
\end{tabular}

\section{Energy reception characteristics varies with date and latitude}

First of all, this paper analyses the changes of energy reception characteristics of the Tropics of Cancer and Capricorn in a year. In the calculation, the latitude north is positive, the south is negative, and the range of change is $\Phi=[-23.5,23.5]^{\circ}$; The range of dates is $d_{n}=$ $[1,365]$; The geographical longitude is $L=110^{\circ}$. In addition, the energy reception characteristics of spring equinox $d_{n}=80$, summer solstice $d_{n}=173$, autumnal equinox $d_{n}=266$ and winter solstice $d_{n}=356$ are compared and analysed. 

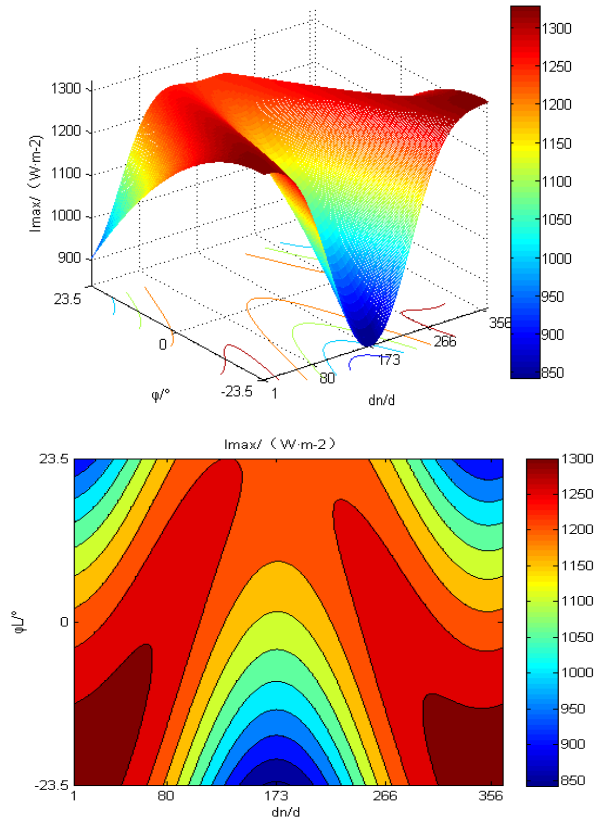

Figure 1. Maximum solar radiation intensity $I_{\max }$ varies with date $d_{n}$ and latitude $\Phi$.
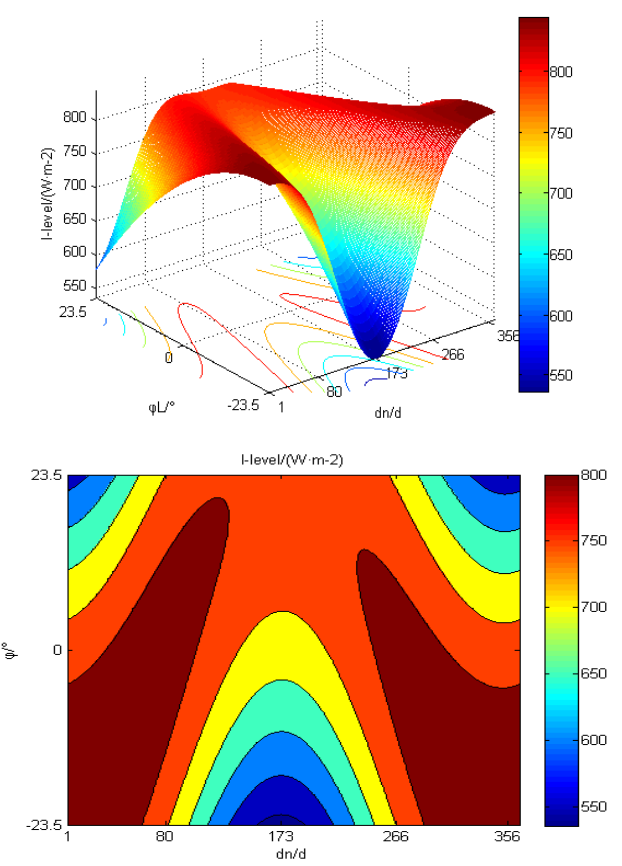

Figure 2. Daytime average solar radiation intensity $I_{\text {level }}$ varies with date $d_{n}$ and latitude $\Phi$.
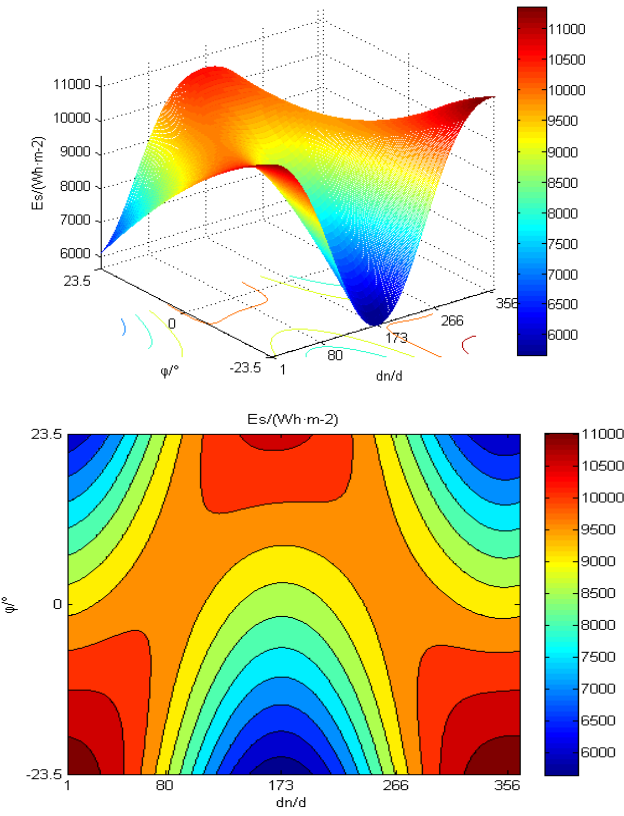

Figure 3. The total amount of solar radiation received per unit area $E_{\mathrm{s}}$ in one day varies with date $d_{n}$ and latitude $\Phi$.
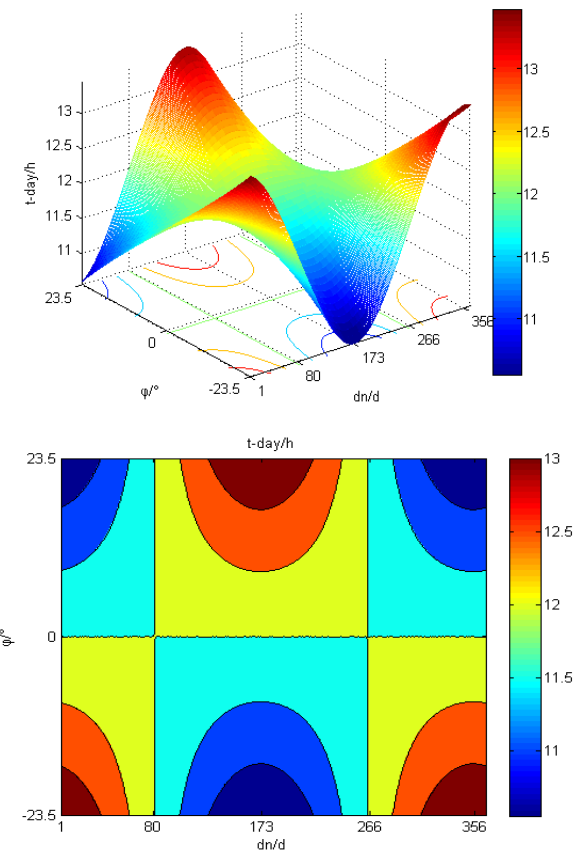

Figure 4. Daytime hours $t_{\text {day }}$ varies with date $d_{n}$ and latitude $\Phi$

Table 2. The maximum and minimum of $I_{\mathrm{max}}, I_{\mathrm{level}}, E_{\mathrm{s}}$ and $t_{\mathrm{day}}$ and the corresponding $\Phi$ and $d_{n}$

\begin{tabular}{|c|c|c|c|c|c|c|c|c|c|}
\hline & \multicolumn{4}{|c|}{ minimum } & \multicolumn{4}{|c|}{ maximum } \\
\hline & & $\begin{array}{l}I_{\max } \\
(\mathrm{W} / \\
\left.\mathbf{m}^{2}\right)\end{array}$ & $\begin{array}{c}I_{\text {level }} \\
\left(\mathrm{W} / \mathrm{m}^{2}\right)\end{array}$ & $\begin{array}{c}E_{\mathbf{s}} \\
(\mathbf{W h} / \\
\left.\mathbf{m}^{\mathbf{2}}\right)\end{array}$ & (h) ${ }^{t_{\text {day }}}$ & $\begin{array}{l}I_{\max } \\
(\mathrm{W} / \\
\left.\mathbf{m}^{2}\right)\end{array}$ & $\left(\mathrm{W} / \mathrm{m}^{2}\right)^{I_{\text {level }}}$ & $\begin{array}{c}E_{\mathbf{s}} \\
(\mathbf{W h} / \\
\left.\mathbf{m}^{\mathbf{2}}\right)\end{array}$ & (h) ${ }^{t_{\text {day }}}$ \\
\hline \multirow{3}{*}[0,23.5]{$^{\circ}$} & value & 898.7 & 572.13 & 6036.32 & 10.55 & 1294.63 & 824.18 & 10633.76 & 13.45 \\
\hline & $\Phi /^{\circ}$ & 23.5 & 23.5 & 23.5 & 23.5 & 0 & 0 & 23.5 & 23.5 \\
\hline & $d_{n} / \mathbf{d}$ & 354 & 354 & 354 & 355 & 70 & 70 & 168 & 172 \\
\hline \multirow{3}{*}{$\begin{array}{c}{[-} \\
23.5,0]^{\circ}\end{array}$} & value & 842.15 & 536.13 & 5656.45 & 10.55 & 1325.73 & 843.99 & 11346.79 & 13.45 \\
\hline & $\Phi /^{\circ}$ & -23.5 & -23.5 & -23.5 & -23.5 & -23 & -23 & -23.5 & -23.5 \\
\hline & $d_{n} / \mathrm{d}$ & 173 & 173 & 173 & 172 & 365 & 365 & 357 & 355 \\
\hline
\end{tabular}


figure 1 and figure 2 are the curves of $I_{\max }$ and $I_{\text {level }}$ with date $d_{n}$ and latitude $\Phi$. The changes in figure 1 and figure 2 are basically the same. Near the Tropic of Cancer, the double peaks of $I_{\max }$ and $I_{\text {level }}$ appear in the vicinity of the spring equinox and the autumnal equinox, and fall in the summer solstice, and the trough value appears near the winter solstice; Near the Tropic of Capricorn, the peaks of $I_{\max }$ and $I_{\text {level }}$ appear near the winter solstice, and the trough value appears near the summer solstice, showing a relatively perfect sinusoidal trend.

figure 3 and figure 4 are the curves of $E_{\mathrm{s}}$ and $t_{\text {day }}$ with date $d_{n}$ and latitude $\Phi$. The change trend of figure 3 and figure 4 are basically the same. In the vicinity of the tropic, both $E_{\mathrm{s}}$ and $I_{\text {level }}$ show a relatively perfect sinusoidal trend. Near the Tropic of Cancer, the peak value appears near the summer solstice, and the trough value appears near the winter solstice; And it's opposite near the Tropic of Capricorn. In addition, figure 4 shows that the day and night time are equal at the vernal equinox and autumnal equinox between the Tropics of Cancer and Capricorn. At the equator, the time of day and night are the same all year round. This is consistent with the actual situation, and it verifies the reliability and accuracy of the calculation model to a certain extent.

Table 2 shows the maximum and minimum values and the corresponding latitude and date of the energy reception characteristics between the Tropic of Cancer and the equator $[0,23.5]^{\circ}$, the equator and the Tropic of Capricorn $[-23.5,0]^{\circ}$.

\section{Energy reception characteristics varies with longitude and latitude}

On the basis of the above analysis, this paper analyses the changes of solar energy reception characteristics between the Tropics of Cancer and Capricorn in a day with longitude and latitude. When calculating, the spring equinox day $d_{n}=80$ is chosen as the research object. The latitude range is $\Phi=[-23.5,23.5]^{\circ}$, and the range of longitude is $L=[105,118]^{\circ}$.
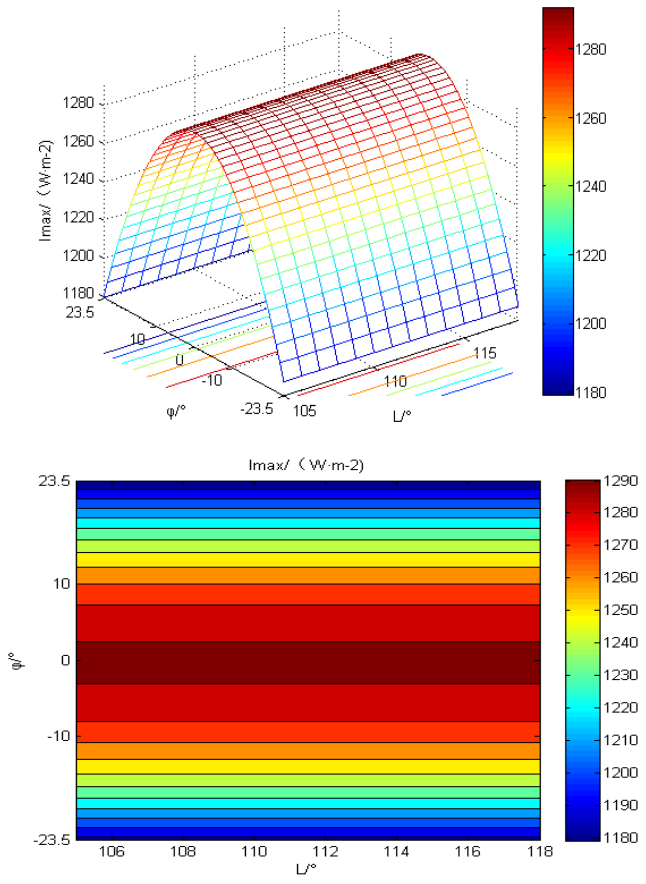

Figure 5. Maximum solar radiation intensity $I_{\max }$ varies with longitude $L$ and latitude $\Phi$.
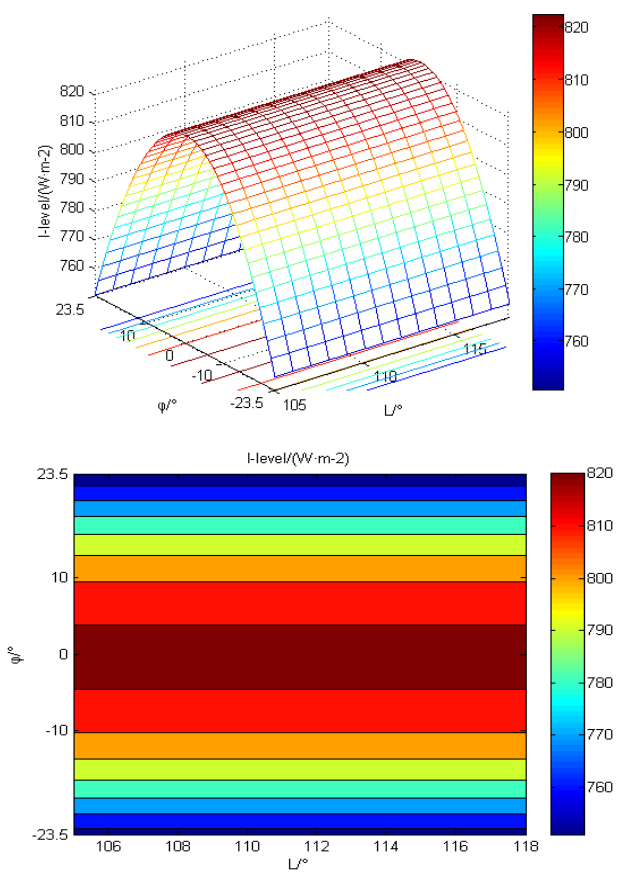

Figure 6. Daytime average solar radiation intensity $I_{\text {level }}$ varies with longitude $L$ and latitude $\Phi$. 

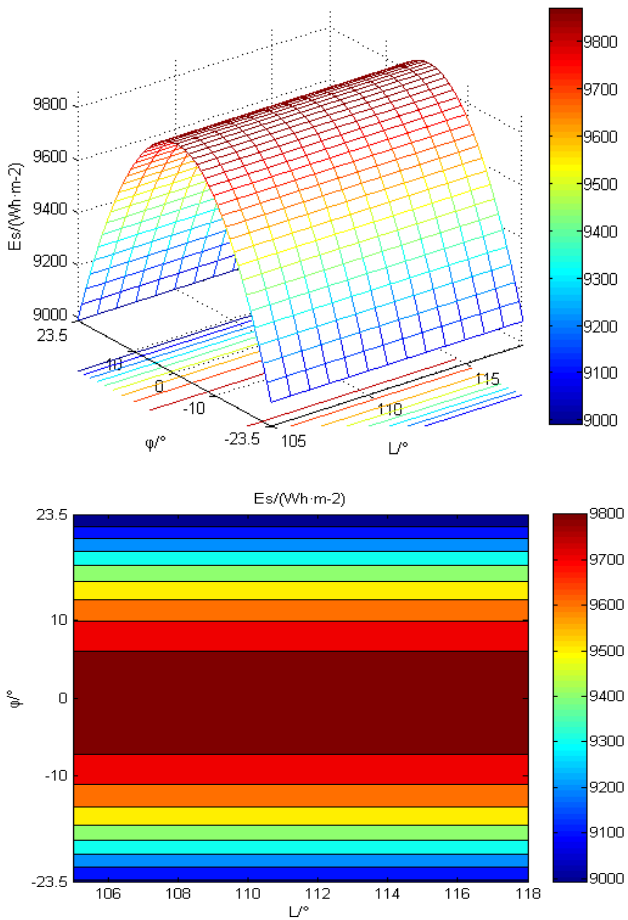

Figure 7. The total amount of solar radiation received per unit area $E_{\mathrm{s}}$ in one day varies with longitude $L$ and latitude $\Phi$.
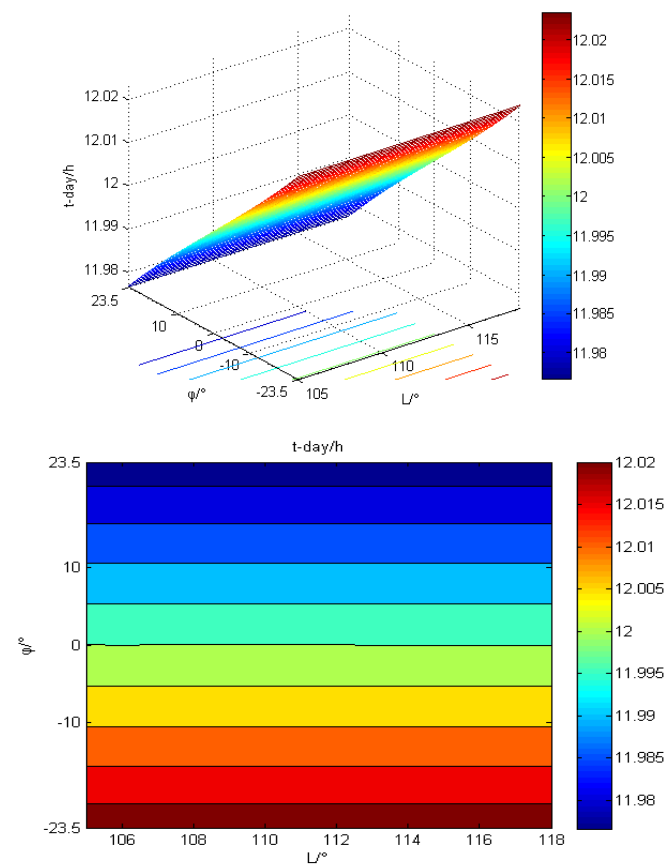

Figure 8. Daytime hours $t_{\text {day }}$ varies with longitude $L$ and latitude $\Phi$.

Figure 5-8 shows the variation curves of $I_{\max }, I_{\text {level}}$, $E_{\mathrm{s}}$ and $t_{\mathrm{day}}$ with longitude $L$ and latitude $\Phi$ at vernal equinox. It can be seen that the $I_{\max }, I_{\text {level }}, E_{\mathrm{s}}$ and $t_{\text {day }}$ in a day don't vary with longitude, but changes with latitude. On the vernal equinox day, the $I_{\max }, I_{\text {level }}$ and $E_{\mathrm{s}}$ are sinusoidal between the Tropics of Cancer and Capricorn, and the maximum values are obtained near the equator. The length of the day is around $12 \mathrm{~h}$ in this area.

\section{Conclusion}

In this paper, the energy reception characteristics including $I_{\text {max }}, I_{\text {level }}, E_{\mathrm{s}}$ and $t_{\text {day }}$ of $20000 \mathrm{~m}$ high altitude area between the Tropics of Cancer and Capricorn are calculated with the change of date $d_{n}$ and latitude $\Phi$ within one year, and with the longitude $L$ and latitude $\Phi$ within one day (vernal equinox).

Through analysis, it can be seen that:

1) In a year, the energy reception characteristics including $I_{\text {max }}, I_{\text {level }}, E_{\mathrm{s}}$ and $t_{\text {day }}$ of a certain longitude will vary with the date and latitude;

2) In a day, the energy reception characteristics including $I_{\text {max }}, I_{\text {level }}, E_{\mathrm{s}}$ and $t_{\text {day }}$ only change with latitude, not with longitude.

Solar radiation is abundant in the near space between the Tropics of Cancer and Capricorn. In this paper, the energy reception characteristics of solar aircraft are analysed, and the corresponding calculation results are given. In particular, the maximum and minimum values of energy reception characteristics can provide some reference for the design of solar aircraft for different flying missions in the region.

\section{References}

1. H.T. Sang, D.(Lanzhou Traffic University) (2016)

2. W.T. Hu, D.(Tianjin University) (2013)

3. X.Z. Gao, D.(National University of Defense Technology) (2014)

4. X.D. Mei, J.L. Sun, Z.Q. Li, X.F. Chen, J. Xing, H. $\mathrm{Xu}$, L.L. Qie, Y. Lv, M. Li, and L. Li, Spectroscopy and Spectral Analy. 36(03), 609-617 (2016)

5. Y. Zhou, China Mod. Edu. Equ. 20, 44-45 (2013)

6. S. Huang, A Vast View on Publishing, 01, 38-39 (2015)

7. C.P. Lu, K. Ji, and F. Wang, Tech. Wind, 24, 62 (2015)

8. J. Yang, Z.Z. Liu, B. Meng, and N. Li, Energy Eng. 01, 35-38 (2011)

9. L.J. Ding, H.Y. Li, Ningxia Engin. Tech. 14(01), 79,15 (2015) 